\title{
HIGH ENERGY HADRON-HADRON COLLISIONS
}

\author{
Annual Progress Report
}

T. T. Chou

Physics Department

University of Georgia

Athens, Georgia 30602

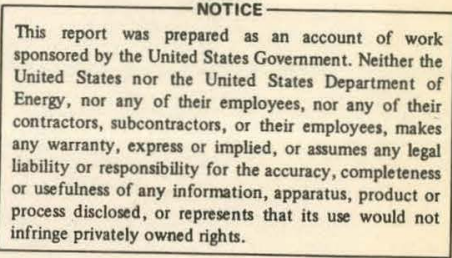

Date Submitted - October 1977

PREPARED FOR THE

U.S. ENERGY RESEARCH AND DEVELOPMENT ADMINISTRATION

UNDER CONTRACT EY-76-S-09-0946 


\section{DISCLAIMER}

This report was prepared as an account of work sponsored by an agency of the United States Government. Neither the United States Government nor any agency Thereof, nor any of their employees, makes any warranty, express or implied, or assumes any legal liability or responsibility for the accuracy, completeness, or usefulness of any information, apparatus, product, or process disclosed, or represents that its use would not infringe privately owned rights. Reference herein to any specific commercial product, process, or service by trade name, trademark, manufacturer, or otherwise does not necessarily constitute or imply its endorsement, recommendation, or favoring by the United States Government or any agency thereof. The views and opinions of authors expressed herein do not necessarily state or reflect those of the United States Government or any agency thereof. 


\section{DISCLAIMER}

Portions of this document may be illegible in electronic image products. Images are produced from the best available original document. 
This report was prepared as an account of work sponsored by the United States Government. Neither the United States nor the United States Energy Research and Development Administration, nor any of their employees, nor any of their contractors, subcontractors, or their employees, makes any warranty, express or implied, or assumes any legal liability or responsibility for the accuracy, completeness or tusefulness of any information, apparatus, product 3 or process disclosed, nor represents that its use would not infringe privately owned rights. 


\section{ABSTRACT}

Work on high energy hadron collisions in the geometrical picture, performed under the ERDA Contract No. E(38-1)-946, is summarized. Comparisons with new experimental results are made. Specific items discussed include (a) the hadronic matter distribution for mesons and the comparison of theoretical prediction with a recent experiment, (b) the possible existence of a second minimum in elastic pp scattering at. very high energies, (c) the analysis of the energy dependence of opaqueness in pp collisions, and (d) the study of spin effects in high energy polarized Kd elastic scattering. 
(I) Introduction

Experimental results from ISR and FNAL during the past few year indicate that the geometrical picture of hadron collisions is extremely useful. This picture ${ }^{1,2}$ was proposed to accomodate many of the striking characteristics and regularities observed in high energy collisions, such as the existence ${ }^{3}$ of diffraction phenomena in elastic processes, and the $1 \mathrm{ack}{ }^{4}$ of large transverse momenta in inelastic processes. The basic idea which underlies the geometrical approach is that the hadrons are extended objects consisting of an infinite number of constituents. In terms of the hadronic matter distributions, a model ${ }^{5}$ for elastic scattering was formulated. This theory yielded several predictions which were remarkably confirmed later by experiments. Pursuance of the geometrical ideas led naturaily to conclusions about inelastic processes (the hypothesis of limiting fragmentation ${ }^{6}$ ), and generated other new ideas and concepts ${ }^{7-10}$ useful in high energy collisions. We shall not review the geometrical approach here in any detail as a summary of the results for this approach were outlined in the previous proposal. In the following we describe the results of our recent work based on the geometrical picture of hadron collisions, especially those performed under the ERDA contract during the past year. The relations between our work and some new experiments are also discussed. 
(II) Hadronic matter distribution for mesons

The geometrical model of elastic scattering relates 5 hadron-hadron and electron-hadron collisions at very high energies. A no-parameter calculation using experimental pp elastic data as input yielded the hadronic matter distribution for the proton and the results are in good agreement 11,12 with the experimental proton electric form factor measured in ep scattering. Utilizing the Fermilab $200 \mathrm{GeV}$ data ${ }^{13}$ on $\pi^{ \pm} \mathrm{p}$ and $K^{ \pm}$p collisions we also predicted ${ }^{14}$ the form factors for pions and kaons and their root mean square radii. Confrontation with experiment before 1976 was not possible because previous measurements of the pion radius had produced data with very large uncertainties. No attempt to measure kaon radius had been made. However, last year the UCLA-Notre Dame-Pittsburg-FNAL-Dubna Collaboration successfully carried out a precise measurement at FNAL on the meson form factors. This was done by scattering high energy mesons off atomic electrons. Their preliminary data on the pion radius, reported ${ }^{15}$ at the Tbilisi conference, agreed well with our predicted value. The remarkable agreement further indicates that the geometrical description of hadronic structure is accurate and useful. Data gathered in the Kaon experiment is still being analyzed and results are expected to be available in a few months. Comparison of our kaon form factor prediction with experiment is anticipated with interest. (III) Structures in pp differential cross"sections at very high energies The geometrical picture has had impressive successes in 
elastic pp scattering. (a) It produced excellent agreement ${ }^{16}$ with high energy pp differential cross section, down to details, in $-t<1.2(\mathrm{GeV})^{2}$ region. (b) It predicted 17,18 the existence of the first minimum and the second maximum at higher -t values, which were later found in CERN-ISR experiments. ${ }^{19}$ The agreement of the calculation ${ }^{16}$ with experimental data around the first minimum and the second maximum is very good. (c) since the total cross section increases with increasing energy, the calculation predicts ${ }^{20}$ a shift of the position of the first minimum with energy and a concurrent rise of the second maximum. These characteristics were later found to be in agreement with experiment ${ }^{21}$. However the picture also predicts ${ }^{17,18}$ a second mimimum and a third maximum which has so far not been experimentally verified.

Recently there have been published 22,23 new data for high $t$, high energy elastic pp scattering. It has been observed 22,23 that the slope parameter is smaller at higher $t$ values. An additional characteristic that we noted (Report \#2) is that the trends of change of the experimental slope parameter with energy in the low $t$ and high $t$ regions seem to proceed in opposite directions. If this trend continues into higher energies the differential cross section curve must "cave in" in the $-t=4$ to $6(\mathrm{GeV})^{2}$ region. Therefore we predicted ${ }^{24}$ at energies much above $2000 \mathrm{GeV}$ structures in the form of a shoulder, or a minimum and maximum, will develop in this $t$ region just as the geometrical model calculation 17,18 has indicated. Our conjecture is best tested with future acceler- 
ators such as the ISABELLE. Some preliminary tests of our speculation in the ISR energy range can also be made and was discussed in the paper (Report \#2).

(IV) Energy dependence of opaqueness in pp collisions

since the rising total cross section was conjectured ${ }^{25}$ by Cheng and Wu and subsequently confirmed 26 at ISR, much discussion has been centered on the behavior of pp opaqueness at very high energies. The opaqueness evaluated from experimental data could provide important guidelines for the development of elastic models. On the theoretical side there exist two distinctly different proposals for incorporating energy dependence into elastic scattering models, the factorizable eikonal model 20, 27 (which is a natural extension of the geometrical picture) and the model of geometrical scaling ${ }^{28}$ (not related to the geometrical picture). The former has much intuitive appeal as it. allows a physical interpretation for the b-dependence of the opaqueness. However, the scaling model still lacks such an interpretation. Nevertheless, the validity of theoretical proposals must be decided by experiment. Tests are now made possible by the newer and more complete pp data ${ }^{21}$ available from ISR. Our preliminary analysis ${ }^{29}$ of the CHOV experiment shows (Report \#1) that the opaqueness increment between two extreme ISR energies is more central than the opaqueness itself. This result is consistent with factorization but incompatible with scaling. Later a more extensive parametrization-independent impact parameter studies (Report \#3), taking into account the real part effect and including a complete error analysis, 
reaffirmed ${ }^{30}$ our previous conclusions. Therefore we now believe that eikonal models with factorizable opaqueness are very likely valid, but the determination of the precise form for the energy dependent factor will require more accurate data taken at several different energies.

(V) Hadronic matter current distribution inside a polarized hadron and a polarized nucleus

In the original geometrical description ${ }^{5}$ of the structure of hadrons, each particle was regarded as an extended object with a hadronic matter density distribution, but the possible motion of hadronic matter inside a hadron was ignored. The theory was generalized ${ }^{31}$ in 1976 to include the effect of the internal motion of hadronic matter, thus the concept of hadronic matter current distribution for polarized hadrons was introduced. The increasing opaqueness of hadrons relative to each other at increasing relative velocities allows the matter current to be obtained from a measurement of the spin rotation parameter $R(t)$ in elastic hadron-hadron scattering. The spin dependence in elastic hadron-hadron scattering is solely due to this non-vanishing $R$ parameter at very high energies. Other spin effects, which might also contribute to the value of $R$ at lower energies, disappear in the high energy region. Based on the hypothesis that the electric charge-current distribution and the hadronic matter density-current distribution for the proton are proportional, numerical estimates of the magnitude of $\mathrm{R}$ for $\mathrm{Kp}, \pi \mathrm{p}$ and $\mathrm{pp}$ collisions were made. Results indicated that the spin effect arising from the velocity distribution 
within the proton is indeed measurable with available techniques. There now exist at least two experimental groups 32,33 at ANL and CERN interested in making precise measurements of the spin rotation effect. If their experimental proposals are approved, the validity of our theory could be tested in about two years.

In order to elucidate the fundamental ideas, the original papers 10,31 on velocity profile discussed only the case of spin one-half hadrons. The generalization to higher spins presents no special difficulties in principle. In Report \#4 (in preparation), the spin effects in the $\pi d$ collision are estimated, where the deuteron is treated as a spin-one elementary particle with its matter density-current distribution obtained from nuclear theory. Similar studies for other nuclei would also be very useful and will be carried out later. Such predictions may be compared with future experiments and they can provide checks on existing nuclear theories. In general, studies of the matter current effect on hadrons and nuclei could provide considerable insight into their structures. Further work along this line is clearly warranted. 


\section{References}

1. T. T. Wu and C. N. Yang, Phys. Rev. 137, B 708 (1965).

2. N. Byers and C. N. Yang, Phys. Rev. 142, 976 (1966).

3. K. J. Foley et al., Phys. Rev. Letters 10, 376 (1963); 11, 425 (1963).

4. See, for example, 0. Czyzewski, in 14th International Conference on High Energy Physics (Vienna, 1968), p. 367.

5. T. T. Chou and C.N. Yang, in High Energy Physics and Nuclear Structure, edited by G. Alexander (North-Holland Publishing Co., Amsterdam, 1967), pp. 348-359; T. T. Chou and C. N. Yang, Phys. Rev. 170, 1591 (1968).

6. J. Benecke, T. T. Chou, C. N. Yang, and E. Yen, Phys. Rev. $\underline{188}, 2159$ (1969).

7. T. T. Chou and C. N. Yang, Phys. Rev. Letters 25, 1072 (1970) 。

8. T. T. Chou, Phys. Rev. Letters 27, 1247 (1971).

9. T. T. Chou and C. N. Yang, Phys. Rev. D7, 1425 (1973).

10. T. T. Chou, in High Energy Collisions - 1973, edited by C. Quigg (AIP, New York, 1973), pp. 118-123.

11. A. W. Chao and C. N. Yang, Phys. Rev. D8, 2063 (1973).

12. V. Franco, Phys. Rev. D11, 1837 (1975).

13. C. W. Akerlof et al., Phys. Rev. Dl4, 2864 (1976).

14. '1'. T. Chou, Phys. Rev. D11, 3145 (1975).

15. E. Dally et al., paper submitted to Tbilisi Conference (1976).

16. M. Kac, Nucl. Phys. B62, 402 (1973). :

17. T. T. Chou and C. N. Yang, Phys. Rev. Letters 20, 1213 (1968).

18. L. Durand and R. Lipes, Phys. Rev. Lett. 20, 637 (1968). 
19. A. Böhm et al., Phys. Letters 49B, 491 (1974).

20. F. Hayot and U. P. Sakhatme, Phys. Rev. D10, 2183 (1974)..

21. N. Kwak et al., Phys. Lett. 58B, 233 (1975).

22. H. De Kerret et al., Phys. Lett. $62 \mathrm{~B}, 363$ "(1976); H. De Kerret et al., Phys. Lett. 68B, 374 (1977).

23. J. L. Hartmann et al., Phys. Rev. Lett. (to be published).

24. T. T. Chou and C. N. Yang, (submitted to Phys. Rev. Lett. for publication).

25. H. Cheng and T. T. Wu, Phys. Rev. Lett, 24, 1456 (1970).

26. U. Amaldi et al., Phys. Lett, 44B, 112 (1973).

27. R. Henzi, B. Margolis and P. Valin, Phys. Rev. Lett. 32, 1077 (1974); H. Cheng, J. K. Walker and T. T. Wu, Phys. Lett. $\underline{4 \mathrm{~B}}, 97$ (1973).

28. A. J. Buras and J. Dias de Deus, Nucl. Phys. B71, 481 (1974). 29. T. T. Chou, (to be published in Foundations of Physics). 30. T. T. Chou, (submitted to Nucl. Phys. for publication). 31. T. T. Chou and C. N. Yang, Nucl. Phys. B107, I (1976). 32. A. Yokosawa, private communication. 33. K. Kuroda, private communication. 